\title{
Measuring the true supernova rate in galaxies
}

\author{
Mark R. Kidger and Fabiola Martín-Luis \\ Instituto de Astrofísica de Canarias, \\ c/vía Láctea s/n, E-\$8205 La Laguna, Tenerife, España
}

\begin{abstract}
One of the best tracers of massive stars is the rate of supernovae in galaxies. Studies of the supernova rate in galaxies are though severely affected by the line of sight extinction. Geometrical effects (e.g., edgewise presentation) may lead to the majority of supernovae that appear in a galaxy being undetectable in the visible. We propose to use Canaricam on the Spanish $10 \mathrm{~m}$ Gran Telescopio Canarias to measure the true supernova rate in a sample of galaxies, independent of extinction and line of sight effects, by measuring at $10 \mu \mathrm{m}$ where the extinction is almost negligible. This system allows Type Ia SNe to be detected out to $100 \mathrm{Mpc}$ in $120 \mathrm{~s}$ of integration, thus 360 galaxies per year for which we are sensitive to SNe can be covered per hour of telescope time per month.
\end{abstract}

\section{Introduction}

As supernovae are the end products of massive stars, they are both tracers of star formation and of the most massive stars in galaxies. Supernovae also determine the chemical evolution of galaxies and are thus of great importance in the study of the star formation rate and galactic evolution. Reliable determination of the SN rate in galaxies is thus a critical parameter, but difficult to measure. Canaricam, the mid-IR imager for the 10.4m Gran Telescopio Canarias, will enter service as a Day 1 instrument in 2004 . It has a $25{ }^{\prime \prime} 4 \times 19 .^{\prime} 8$ field of view and an extensive suite of broad and narrow-band filters (Kidger 2002). This makes it ideally suited to exploring the inner regions of relatively nearby galaxies. One of the most important selection effects, particularly in spiral galaxies, is that of line of sight extinction. The extinction is greatly reduced at $10 \mu \mathrm{m}$, particularly away from the centre of the strong silicate dust feature allowing deep penetration of dust. At $8 \mu \mathrm{m}$ the extinction is $2 \%$ of $A_{V}$, while at $13 \mu \mathrm{m}$ it is $3 \%$ of $A_{V}$ (Rieke \& Lebofsky 1985). Heavily extinguished supernovae can be detected at these wavelengths, thus the efficiency of detection of SNe will be close to $100 \%$.

\section{Strategy}

Little $10 \mu \mathrm{m}$ photometry of SNe has been carried out due to lack of suitable instrumentation. Knowledge of the mid-IR emission of SNe is limited to SN 1987A, an underluminous Type II. Combining visible (Hamuy et al. 1988) and infrared photometry (Bouchet et al. 1989) shows that the $V-N_{3}$ colour index increases 


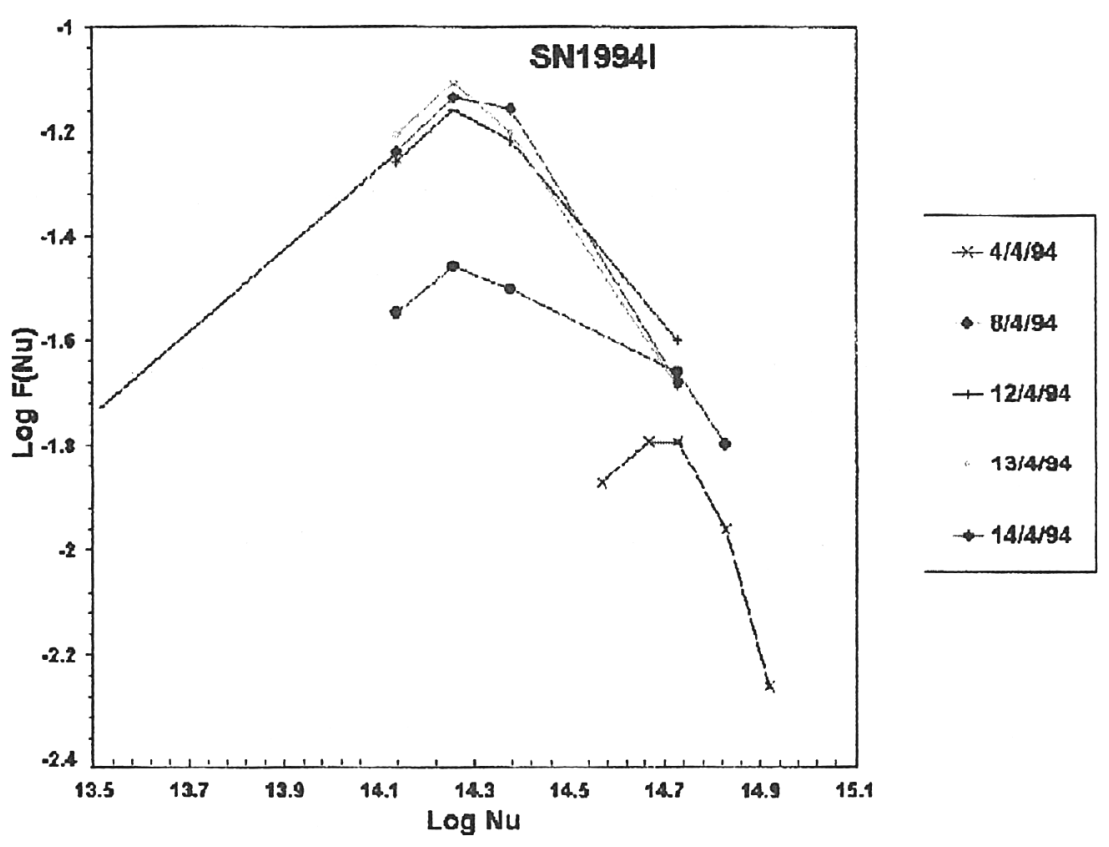

Figure 1. The spectral energy distribution of the Type Ia SN 1994i taken from the literature and unpublished near-IR data from the Carlos Sánchez Telescope at Teide Observatory.

from $+3.2 \mathrm{mag}$ at discovery, to $+6.0 \mathrm{mag}$ as the dust envelope forms. Extrapolation of visible and near-IR photometry of Type I SNe suggests a colour index $V-N_{3} \simeq 5$ (see Figure 1). The sensitivity of CanariCam will thus allow even unreddened SNe to be detected to $V=16$ in 120 s. Given $\mathrm{M}_{V}=-19$ for Type Ia, these can be detected to $>100 \mathrm{Mpc}$, at which distance the field of view of the instrument is $4 \times 10^{4}$ light years. Longer integrations would allow smaller numbers of more distant galaxies that cover the full array to be observed.

Thus a sample of up to 30 galaxies could be observed in $1 \mathrm{hr}$. Given $1 \mathrm{hr}$ of telescope time every 3 nights, 3600 fields could be covered in a year, allowing multiple observations to be made of several hundred galaxies.

\section{References}

Bouchet, P., Slezak, E., Le Bertre, T., Moneti, A., Manfroid, J. 1989, A\&AS 89, 379 Hamuy, M., Suntzeff, N.B., González, R., Martín, G. 1988, AJ 95, 63 Kidger, M.R. 2002, in: Science with the Gran Telescopio Canarias (GTC), RMxAA$\mathrm{SC}$ in press

Rieke, G.H., Lebofsky, M.J. 1985, ApJ 288, 618 